\title{
Optimal Unravellings for Feedback Control in Linear Quantum Systems
}

\author{
H. M. Wiseman ${ }^{1}$ and A. C. Doherty ${ }^{2}$ \\ ${ }^{1}$ Centre for Quantum Computer Technology, Centre for Quantum Dynamics, \\ School of Science, Griffith University, Brisbane 4111 Australia \\ ${ }^{2}$ School of Physical Sciences, University of Queensland, Brisbane 4072 Australia
}

(Dated: 24th November, 2004)

\begin{abstract}
For quantum systems with linear dynamics in phase space much of classical feedback control theory applies. However, there are some questions that are sensible only for the quantum case, such as: given a fixed interaction between the system and the environment what is the optimal measurement on the environment for a particular control problem? We show that for a broad class of optimal (state-based) control problems (the stationary Linear-Quadratic-Gaussian class), this question is a semi-definite program. Moreover, the answer also applies to Markovian (current-based) feedback.

PACS numbers: 03.65.Yz, 02.30.Yy, 42.50.Lc
\end{abstract}

Classical feedback control is a large and well established area of engineering [1, 2]. Quantum feedback control, by contrast, is still rapidly developing (see Ref. [3] for a recent review of theory) especially with the notable success of recent experiments in the field [4, 5, 6, 7]. Although problems in quantum control are generally quite distinct from their classical counterparts, for quantum systems with linear dynamics in phase space, and with Gaussian noise, classical optimal feedback control theory can be applied. This idea was introduced by Belavkin [8], and its physical application was developed in Refs. 9]. In linear systems the key differences between the quantum and classical problems are the limits resulting from measurement backaction noise. The significance of this field is that in many quantum feedback control experiments a linear theory can be used, for example in quantum optics [5, 10, atomic ensembles [6, 11], nanomechanics [7, 12] and cavity QED [13].

This Letter contains several important advances in this field. First, we give a completely general theory of feedback control in linear quantum systems with a fixed coupling to the environment, allowing for arbitrary unravellings (ways of monitoring the environment). Second, having formulated the conditional evolution under arbitrary unravellings as stochastic differential equations for the moments, we show that there is a stabilizing solution $W$ for the conditioned covariance matrix under weaker conditions than classically. Third, we identify which unconditional evolutions allow for such a $W$ to exist for almost all unravellings, and show that the set of all possible $W \mathrm{~s}$ is set by two linear matrix inequalities (LMIs). Fourth, we show that for all stationary problems with a cost function that is quadratic in the system and controller variables, the optimal unravelling for optimal feedback control can be found efficiently using a semidefinite program. Fifth, we show that if all control constraints are removed, Markovian feedback control (which is much simpler both conceptually and experimentally) performs identically to optimal feedback control. We conclude with an example followed by discussion.
Continuous Markovian Unravellings. - The most general autonomous differential equation for the state of a quantum system is the Lindblad master equation

$$
\hbar \dot{\rho}=-i[\hat{H}, \rho]+\sum_{l=1}^{L} \mathcal{D}\left[\hat{c}_{l}\right] \rho \equiv \mathcal{L}_{0} \rho
$$

Here $\hat{H}=\hat{H}^{\dagger}$ is the system Hamiltonian, $\left\{\hat{c}_{l}\right\}$ is a set of operators that are arbitrary (although for rigour 14, 15. they should be bounded). The action of $\mathcal{D}[\hat{c}]$ on an arbitrary operator $\rho$ is defined by

$$
\mathcal{D}[\hat{c}] \rho \equiv \hat{c} \rho \hat{c}^{\dagger}-\left(\hat{c}^{\dagger} \hat{c} \rho+\rho \hat{c}^{\dagger} \hat{c}\right) / 2 .
$$

Lindblad master equations are widely used in atomic, optical, and nuclear physics 16. They can typically be derived if the system is coupled weakly to an environment that is large (i.e. with dense energy levels). Because the system typically becomes entangled with the environment, tracing over the latter typically leads to loss of system purity. However, there is no necessity to ignore the environment. Indeed, under the conditions that allow the derivation of a Lindblad master equation, it is possible to measure the environment continually on a time scale much shorter than any system time of interest. This monitoring yields information about the system, producing a stochastic conditioned system state $\rho_{\mathrm{c}}$ that on average reproduces the unconditioned state $\rho$. That is, the master equation is unravelled into stochastic quantum trajectories 18], with different measurements on the environment leading to different unravellings.

If we ask that the monitoring also yield an evolution for $\rho_{\mathrm{c}}$ that is, like that of Eq. (11). continuous and Markovian, then it must be of the form 19

$$
\hbar d \rho_{\mathrm{c}}=\mathcal{L}_{0} \rho_{\mathrm{c}} d t+d \mathbf{z}^{\dagger}(t) \Delta_{\mathrm{c}} \hat{\mathbf{c}} \rho_{\mathrm{c}}+\rho_{\mathrm{c}} \Delta_{\mathrm{c}} \hat{\mathbf{c}}^{\dagger} d \mathbf{z}(t) .
$$

Note that here the $\dagger$ indicates transpose $(T)$ of the vector and Hermitian adjoint of its components, and $\hat{\mathbf{c}}=\left(\hat{c}_{1}, \cdots, \hat{c}_{L}\right)^{\top}$. We are also using the notation $\Delta_{\mathrm{c}} \hat{o} \equiv \hat{o}-\langle\hat{o}\rangle_{\mathrm{c}}$, where $\langle\hat{o}\rangle_{\mathrm{c}} \equiv \operatorname{Tr}\left[\rho_{\mathrm{c}} \hat{o}\right]$, and we have introduced $d \mathbf{z}=\left(d z_{1}, \cdots, d z_{L}\right)^{\top}$ of infinitesimal complex 
Wiener increments [20]. It satisfies $\mathrm{E}[d \mathbf{z}]=0$, where $\mathrm{E}$ denotes expectation value, and has the correlations

$$
d \mathbf{z} d \mathbf{z}^{\dagger}=\hbar \Theta d t, \quad d \mathbf{z} d \mathbf{z}^{\top}=\hbar \Upsilon d t
$$

Here we have generalized Ref. [19] to allow for inefficient detection by introducing a matrix $\Theta$. It is convenient to combine $\Upsilon$ and $\Theta$ in an unravelling matrix

$$
U \equiv \frac{1}{2}\left(\begin{array}{cc}
\Theta+\operatorname{Re}[\Upsilon] & \operatorname{Im}[\Upsilon] \\
\operatorname{Im}[\Upsilon] & \Theta-\operatorname{Re}[\Upsilon]
\end{array}\right)
$$

The set $\mathfrak{U}$ of valid $U_{\mathrm{S}}$ is defined by $U \geq 0, \Upsilon=\Upsilon^{\top}$, and $\Theta=\operatorname{diag}\left(\theta_{1}, \cdots, \theta_{L}\right)$, where $\theta_{l} \in[0,1]$. This can be derived using the method of Ref. [19] by splitting each output channel $l$ into a portion $\theta_{l}$ that is observed and a portion $1-\theta_{l}$ that is unobserved. In the case of efficient monitoring $(\Theta=I)$, Eq. (3) preserves purity and so can be replaced by a stochastic Schrödinger equation [18, 19].

The measurement results upon which the evolution of $\rho_{\mathrm{c}}$ is conditioned is a vector of complex functions

$$
\mathbf{J}^{\top} d t=\left\langle\hat{\mathbf{c}}^{\top} \Theta+\hat{\mathbf{c}}^{\dagger} \Upsilon\right\rangle_{\mathrm{c}} d t+d \mathbf{z}^{\top}
$$

In optics measurements like this (e.g. homodyne detection) give rise to photocurrents, so we call $\mathbf{J}$ a current.

Linear Systems. - We are interested in systems of $N$ degrees of freedoms, with the $n$th described by the canonically conjugate pair obeying the commutation relations $\left[\hat{q}_{n}, \hat{p}_{n}\right]=i \hbar$. Defining a vector of operators

$$
\hat{\mathbf{x}}=\left(\hat{q}_{1}, \hat{p}_{1}, \ldots, \hat{q}_{N}, \hat{p}_{N}\right)^{\top},
$$

we can write $\left[\hat{x}_{n}, \hat{x}_{m}\right]=i \hbar \Sigma_{n m}$, where $\Sigma$ is the $(2 N) \times$ $(2 N)$ symplectic matrix

$$
\Sigma=\bigoplus_{n=1}^{N}\left(\begin{array}{cc}
0 & 1 \\
-1 & 0
\end{array}\right)=\Sigma^{*}=-\Sigma^{\top}=-\Sigma^{-1}
$$

For a system with such a phase-space structure it is possible to define a Gaussian state. Like its classical counterparts, it is determined by its mean vector $\langle\hat{\mathbf{x}}\rangle$ and its covariance matrix, which in the quantum case must be symmetrized: $V_{n m}=\left(\left\langle\Delta \hat{x}_{n} \Delta \hat{x}_{m}\right\rangle+\left\langle\Delta \hat{x}_{m} \Delta \hat{x}_{n}\right\rangle\right) / 2$. For such states, a necessary and sufficient condition on the matrix $V$ is the following LMI 21]

$$
V+i \hbar \Sigma / 2 \geq 0
$$

In this Letter we are concerned with linear systems; that is, ones for which $\hat{H}$ is quadratic, and $\hat{\mathbf{c}}$ linear, in $\hat{\mathbf{x}}$ :

$$
\hat{H}=(1 / 2) \hat{\mathbf{x}}^{\top} G \hat{\mathbf{x}}-\hat{\mathbf{x}}^{\top} \Sigma B \mathbf{u}(t), \quad \hat{\mathbf{c}}=\tilde{C} \hat{\mathbf{x}}
$$

where $G$ is real and symmetric and $B$ is real. The second term in $\hat{H}$ is linear in $\hat{\mathbf{x}}$ to ensure a linear map between the time-dependent classical input $\mathbf{u}(t)$ to the system and the output current $\mathbf{J}(t)$. For such a system, the unconditioned master equation (11) has a Gaussian state as its solution, with the following moment equations

$$
\begin{aligned}
d\langle\hat{\mathbf{x}}\rangle / d t & =A\langle\hat{\mathbf{x}}\rangle+B \mathbf{u}(t), \\
d V / d t & =A V+V A^{\top}+D .
\end{aligned}
$$

Here $A=\Sigma\left(G+\operatorname{Im}\left[\tilde{C}^{\dagger} \tilde{C}\right]\right)$ and $D=\hbar \Sigma \operatorname{Re}\left[\tilde{C}^{\dagger} \tilde{C}\right] \Sigma^{\top}$.

For conditional evolution of linear quantum systems it is convenient to recast the complex current $\mathbf{J}$ of Eq. (6) as a real current with uncorrelated noises:

$$
\mathbf{y} \equiv(\hbar U)^{-1 / 2}\left(\begin{array}{c}
\operatorname{Re}[\mathbf{J}] \\
\operatorname{Im}[\mathbf{J}]
\end{array}\right)=C\langle\hat{\mathbf{x}}\rangle+\frac{d \mathbf{w}}{d t} .
$$

Here $C=2(U / \hbar)^{1 / 2} \bar{C}$, where $\bar{C}^{\top} \equiv\left(\operatorname{Re}\left[\tilde{C}^{\top}\right], \operatorname{Im}\left[\tilde{C}^{\top}\right]\right)$, while $d \mathbf{w}$ is a vector of real Wiener increments satisfying $d \mathbf{w} d \mathbf{w}^{\top}=I d t[20]$. The state $\rho_{\mathrm{c}}$ conditioned on $\mathbf{y}(t)$ is found from Eq. (3). For linear systems this conditioned state is Gaussian, and is analogous to the a posteriori probability distribution propagated by the classical Kalman filter equations, with $d \mathbf{w}$ as the innovation 1 .

Taking expectation values and using the Itô calculus [20], Eq. (3) yields the conditional moment equations

$$
\begin{array}{r}
d\langle\hat{\mathbf{x}}\rangle_{\mathrm{c}}=\left[A\langle\hat{\mathbf{x}}\rangle_{\mathrm{c}}+B \mathbf{u}(t)\right] d t+\left(V_{\mathrm{c}} C^{\top}+\Gamma^{\top}\right) d \mathbf{w} \\
\dot{V}_{\mathrm{c}}=A V_{\mathrm{c}}+V_{\mathrm{c}} A^{\top}+D-\left(V_{\mathrm{c}} C^{\top}+\Gamma^{\top}\right)\left(C V_{\mathrm{c}}+\Gamma\right),
\end{array}
$$

where $\Gamma=-(\hbar U)^{1 / 2} S \bar{C} \Sigma^{\top}$, where

$$
S=\left(\begin{array}{cc}
0 & I \\
-I & 0
\end{array}\right) \text {. }
$$

The stochastic term in $d\langle\mathbf{x}\rangle_{\mathrm{c}}$ and the final term in $\dot{V}_{\mathrm{c}}$ describe the conditioning on the measured current $\mathbf{y}$.

Note that the equation for $V_{\mathrm{c}}$ is deterministic and independent of the measurement results. The final term causes a reduction in uncertainty about the system state (that is, a reduction in the eigenvalues of $V_{\mathrm{c}}$ ). If Eq. (15) has a stabilizing solution [2], then all initial conditions will asymptote to the same steady state. We will notate a stabilizing solution $V_{\mathrm{c}}^{\mathrm{ss}}$ as $W_{U}$ to emphasize that it depends upon the unraveling $U$. Considering efficient detection for simplicity, $W_{U}$ is the solution of

$$
0=\Omega W_{U}+W_{U} \Omega^{\top}-W_{U} C^{\top} C W_{U}+E E^{\top} .
$$

Here $\Omega=\Sigma\left[G+\bar{C}^{\top} S(2 U-I) \bar{C}\right]$ is Hamiltonian drift, while $E=\hbar \Sigma C^{\top} / 2$, manifesting the measurement backaction noise resulting from having $\mathbf{y} \propto C \hat{\mathbf{x}}$. For a general classical problem, $\Omega, C$, and $E$ would be unrelated.

A necessary condition for Eq. (17) to have a stabilizing solution is for $(C, \Omega)$ to be detectable [2], which means

$$
C \mathbf{v} \neq 0 \forall \mathbf{v}: \Omega \mathbf{v}=\lambda \mathbf{v} \text { with } \operatorname{Re}(\lambda) \geq 0 .
$$

That is, all the degrees of freedom that are not strictly stable under the drift matrix $\Omega$ contribute to the signal $C \mathbf{x}$. Classically this becomes a sufficient condition 
if we have also $\left(E^{\top}, \Omega^{\top}\right)$ detectable [2]. Quantally, this extra assumption is unnecessary because of back-action. To see this, note that from Eq. (18), $(C, \Omega)$ detectable $\Longrightarrow\left(C \Sigma^{\top}, \Omega \Sigma^{\top}\right)$ detectable by the invertibility of $\Sigma$; then by the symmetry of $\Omega \Sigma^{\top}$ this implies $\left(C \Sigma^{\top}, \Sigma \Omega^{\top}\right)$ detectable, which implies $\left(E^{\top}, \Omega^{\top}\right)$ detectable as desired. That is, unlike the classical case, detectability of $(C, A)$ [which is equivalent to detectability of $(C, \Omega)$ ] is sufficient for $W_{U}$ to be a stabilizing solution of Eq. (15).

Possible Conditional States. - Let the $U=I / 2$ unravelling be detectable [i.e. let $(\bar{C}, A)$ be detectable]. Then the $W_{U} \mathrm{~s}$ that result from detectable unravellings can be shown to be dense in the set $\mathfrak{W}=\left\{W_{U}: U \in \mathfrak{U}, W_{U}<\infty\right\}$ of finite solutions $W_{U}$ to Eq. (17). Moreover if the $U=I / 2$ unravelling is not detectable then no unravelling is, because $\bar{C} \mathbf{v}=\mathbf{0} \Longrightarrow$ $C \mathbf{v}=\mathbf{0}$. Thus, for all practical purposes, any $W_{U} \in \mathfrak{W}$ will be a stabilizing solution if and only if $(\bar{C}, A)$ is detectable (an unravelling-independent condition).

The set $\mathfrak{W}$ can be determined as follows. Note that from the positivity of the final term in Eq. (15),

$$
D+A W_{U}+W_{U} A^{\top} \geq 0
$$

is a necessary condition on $W_{U}$. This condition means that a state with $V_{\mathrm{c}}=W_{U}$ will evolve unconditionally to a state with $V=W_{U}+d t\left(D+A W_{U}+W_{U} A^{\top}\right) \geq W_{U}$, in other words to a convex (Gaussian) combination of states with $V=W_{U}$. By obtaining information from the bath, the system can thus be steered back to a state with $V_{\mathrm{c}}=W_{U}$ [22]. That is, Eq. (19) is also a sufficient condition on $W_{U}$ for the existence of some $U$ which will yield $V_{\mathrm{c}}^{\mathrm{ss}}=W_{U}$, provided that $W_{U}$ is a valid covariance matrix ( $W_{U} \geq 0$ classically, and $W_{U}+i \hbar \Sigma / 2 \geq 0$ quantally). Moreover, given a $W_{U}$ that satisfies the LMIs (19) and (9) (with $V \rightarrow W_{U}$ ), a (not necessarily unique) unravelling $U$ that will generate it can be found by solving

$$
\hbar R^{\top} U R=D+A W_{U}+W_{U} A^{\top},
$$

where $R=2 \bar{C} W_{U} / \hbar+S \bar{C} \Sigma$. This comes from substituting the expressions for $\Omega, C$ and $E$ into Eq. (177).

Optimal Quantum Control. - In feedback control, $\mathbf{u}(t)$ depends on the history of the measurement record $\mathbf{y}(s)$ for $s<t$. The typical aim of control over some interval $\left[t_{0}, t_{1}\right]$ is to minimize the expected value of a cost function [1], the integral of the sum of positive functions of $\mathbf{x}(t)$ and $\mathbf{u}(t)$ for $t_{0}<t<t_{1}$. For any such problem the separation principle holds: the optimal control $\mathbf{u}(t)$ depends only upon the observer's state of knowledge about the system. That is, in the quantum case, the measurement record $\mathbf{y}(s)$ for $t<s$ is irrelevant except in how it determines $\rho_{\mathrm{c}}(t)$. A special case of interest is that of Linear-Quadratic-Gaussian (LQG) control [1]: a linear system with a quadratic cost function and having Gaussian noise. LQG control has the additional property of certainty equivalence: the only property of $\rho_{\mathrm{c}}$ required is $\langle\hat{\mathbf{x}}\rangle_{\mathrm{c}}$. Moreover, the optimal $\mathbf{u}$ is linear in this mean:

$$
\mathbf{u}(t)=-K(t)\langle\hat{\mathbf{x}}\rangle_{\mathrm{c}}(t) .
$$

In this Letter we specialize to the case of timeindependent cost functions, and $\left(t_{1}-t_{0}\right) \rightarrow \infty$. That is, we wish to minimize $m=\mathrm{E}[h]$ in steady state, where

$$
h=\left\langle\hat{\mathbf{x}}^{\top} P \hat{\mathbf{x}}\right\rangle_{\mathrm{c}}+\mathbf{u}^{\top} Q \mathbf{u},
$$

where $P \geq 0$ and $Q \geq 0$. Note that in steady state

$$
\mathrm{E}_{\mathrm{ss}}\left[\left\langle\hat{\mathbf{x}}^{\top} P \hat{\mathbf{x}}\right\rangle_{\mathrm{c}}\right]=\operatorname{tr}\left[W_{U} P\right]+\mathrm{E}_{\mathrm{ss}}\left[\langle\hat{\mathbf{x}}\rangle_{\mathrm{c}}^{\top} P\langle\hat{\mathbf{x}}\rangle_{\mathrm{c}}\right],
$$

just as in classical control. Assuming that $W_{U}$ is stabilizing, $Q^{-1} B$ exists, and $\left(B^{\top}, A^{\top}\right)$ and $\left(P, A^{\top}\right)$ are detectable, there is a stable optimal control law: $K=$ $Q^{-1} B^{\top} Y$ 2]. Here $Y$ is independent of $U$, satisfying $P+A^{\top} Y+Y A=Y B Q^{-1} B^{\top} Y$. The resulting (minimum) cost is [1]

$$
m_{\mathrm{opt}}=\operatorname{tr}\left[Y B Q^{-1} B^{\top} Y W_{U}\right]+\operatorname{tr}[Y D] .
$$

The significance of Eq. (24) is that it makes it explicit that the choice of unravelling $U$ (which determines $W_{U}$ ) affects the cost of the control. Thus we can ask the following: given an open system with dynamics described by the drift $A$ and diffusion $D$ matrices, what is the optimal way to monitor the bath? Note that classically this would be a nonsensical question as the unconditioned evolution described by $A$ and $D$ would not proscribe the measurements that can be made on the system in any way. But for quantum systems $W_{U}$ will be positive definite because there is no measurement without disturbance, and so $m_{\mathrm{opt}} \neq 0$ even for zero control cost $(Q \rightarrow 0)$.

Finding the optimum unravelling $(U)$ is computationally efficient in the system size $N$. This is because at its heart is the semi-definite program 23] of minimizing a linear function of $W_{U}$ (24) subject to the constraints of the LMIs (9) (with $V \rightarrow W_{U}$ ) and (19). The optimum unravelling $U$ is then found from Eq. (20).

Markovian Quantum Control.- If we remove all constraints on the control by making $B$ full rank and letting $Q \rightarrow 0$, Eq. (24) simplifies to

$$
m_{\mathrm{opt}}=\operatorname{tr}\left[P W_{U}\right]
$$

It turns out that the solution to this problem (the optimal $U$ ) is relevant not only for optimal control, but also for Markovian control as introduced by Wiseman and Milburn 24]. Conceptually and experimentally this is a much simpler form of feedback, as it entails making the time-dependent Hamiltonian linear in the instantaneous output $\mathbf{y}(t)$ [rather than the Kalman-filtered output, $\left.\langle\mathbf{x}\rangle_{\mathrm{c}}(t)\right]$. That is, in the context of the linear system,

$$
\mathbf{u}(t)=F(t) \mathbf{y}(t)
$$

Note that $\mathbf{y}(t)$ has unbounded variation, so doing Markovian control is no less onerous than doing optimal control 
with unbounded $K(t)$ as occurs for $Q \rightarrow 0$. With $B$ invertible we can choose $B F=-W_{U} C^{\top}-\Gamma^{\top}$. This makes Eq. (14) deterministic in the limit $t \rightarrow \infty$ :

$$
d\langle\hat{\mathbf{x}}\rangle_{\mathrm{c}}=M\langle\hat{\mathbf{x}}\rangle_{\mathrm{c}} d t
$$

where $M \equiv A-W_{U} C^{\top} C-\Gamma^{\top} C$. This is the generalization of the optimized Markovian feedback strategy identified for 1-dimensional systems in Refs. 24]. As long as $W_{U}$ is stabilizing, $M$ will be strictly stable [2] so that the solution of Eq. (27) will asymptote to $\mathbf{0}$. Thus from Eq. (23) the cost will again be given by Eq. (25), and the optimal unravelling found as above.

Example.- Consider a system with $N=L=1$ described by the master equation

$$
\hbar \dot{\rho}=-i[(\hat{q} \hat{p}+\hat{p} \hat{q}) / 2, \rho]+\mathcal{D}[\hat{q}+i \hat{p}] \rho,
$$

where the output arising from the second term may be monitored. This could be realized in quantum optics as a damped cavity (harmonic oscillator in the rotating frame) containing an on-threshold parametric down converter [18], with $p$ the squeezed quadrature.

In this case we have $\tilde{C}=(1, i)$, so the drift and diffusion matrices evaluate to $A=\operatorname{diag}(0,-2), D=\hbar I$. Writing the conditional steady-state covariance matrix as

$$
W_{U}=\frac{\hbar}{2}\left(\begin{array}{cc}
\alpha & \beta \\
\beta & \gamma
\end{array}\right)
$$

the LMIs (9) and (19) become

$$
\left(\begin{array}{cc}
\alpha & \beta+i \\
\beta-i & \gamma
\end{array}\right) \geq 0, \quad\left(\begin{array}{cc}
1 & -\beta \\
-\beta & 1-2 \gamma
\end{array}\right) \geq 0
$$

Now say the aim of the feedback control is to produce a stationary state where $q=p$ as nearly as possible. A suitable cost function to be minimized is $\left\langle(\hat{q}-\hat{p})^{2}\right\rangle_{\text {ss }}$. That is, ignoring any control costs, we have

$$
P=\left(\begin{array}{cc}
1 & -1 \\
-1 & 1
\end{array}\right), Q \rightarrow 0
$$

In the optics case it is simple to displace the system in its phase space by application of a coherent driving field [18]. That is, we are justified in taking $B$ to be full rank. Furthermore, any quadratic cost function will be minimized for a pure state so we may assume that $\alpha \gamma=$ $1+\beta^{2}$. Thus the $m$ achievable by optimal or Markovian control is $m=\operatorname{tr}\left[P W_{U}\right]=\left[\left(1+\beta^{2}\right) / 2 \gamma+\gamma / 2-\beta\right] \hbar$, constrained only by $0<\gamma \leq\left(1-\beta^{2}\right) / 2$. The minimum is found numerically to be $m \approx 1.12 \hbar$ at $\beta \approx 0.248$ and $\gamma=\left(1-\beta^{2}\right) / 2$. Proceeding as described above, we find $\sqrt{\hbar} C / 2=-\Gamma / \sqrt{\hbar}=U^{1 / 2}$, with the optimal unraveling

$$
U=\left(\begin{array}{cc}
\cos ^{2} \phi & \cos \phi \sin \phi \\
\cos \phi \sin \phi & \sin ^{2} \phi
\end{array}\right) \text { for } \phi \approx 0.278 \pi
$$

Optically, this unravelling corresponds to homodyne detection with $\phi$ being the local oscillator phase. For Markovian feedback, this gives gives the drift matrix

$$
M=A-4 W_{U} U / \hbar-2 U \approx\left(\begin{array}{ll}
-2.94 & -3.50 \\
-1.65 & -3.97
\end{array}\right),
$$

which is strictly stable as required.

To conclude, we have shown that even for quantum systems that are linear (and so have a classical analogue) the constraints of quantum theory affect the basic structure of feedback control problems. In particular, we have formulated a natural question — the optimal unravelling for stationary LQG control problems - that has no classical analogue. Moreover, these constraints also lead to an efficient algorithm to answer this question. This theory applies to any linearizable system with quantum-limited monitoring. No doubt further fundamental aspects of control for such systems still await discovery.

We thank the ARC and the State of Queensland for support, and K. Jacobs and H. Mabuchi for discussions.

[1] O. L. R. Jacobs, Introduction to Control Theory (Oxford University Press, Oxford, 1993)

[2] K. Zhou, J. C. Doyle, and K. Glover, Robust and Optimal Control (Prentice-Hall, New Jersey, 1996).

[3] H. M. Wiseman, S. Mancini, and J. Wang, Phys. Rev. A 66, 013807 (2002).

[4] W. P. Smith et al., Phys. Rev. Lett. 89, 133601 (2002).

[5] M. A. Armen et al., Phys. Rev. Lett. 89, 133602 (2002).

[6] JM Geremia, J. K. Stockton, and H. Mabuchi, Science 304, 270 (2004).

[7] M. D. LaHaye, O. Buu, B. Camarota, and K. C. Schwab, Science 304, 74 (2004).

[8] V. P. Belavkin, in Information, complexity, and control in quantum physics, edited by A. Blaquière, S. Dinar, and G. Lochak (Springer, New York, 1987) ; V. P. Belavkin, Commun. Math. Phys. 146, 611 (1992) .

[9] A. C. Doherty and K. Jacobs, Phys. Rev. A 60, 2700 (1999); A. C. Doherty et al., ibid. 62, 012105 (2000).

[10] H. M. Wiseman, Phys. Rev. Lett. 75, 4587 (1995).

[11] L. K. Thomsen, S. Mancini, and H. M. Wiseman, Phys. Rev. A (Rapid Comm.) 65, 061801 (2002).

[12] A. Hopkins, K. Jacobs, S. Habib, and K. Schwab, Phys. Rev. B 68, 235328 (2003).

[13] D. A. Steck et al., Phys. Rev. Lett. 92, 223004 (2004).

[14] G. Lindblad, Commun. Math. Phys. 48, 199 (1976).

[15] V. Gorini, A. Kossakowski, and E. C. G. Sudarshan, J. Math. Phys. 17, 821 (1976).

[16] Non-Lindblad-form master equations (including integrodifferential equations and non-autonomous differential equations) are also widely used, when the Markov approximation is invalid. For such systems the unravelling of the master equation into stochastic trajectories cannot be interpreted using standard quantum measurement theory — see Ref. [17]. The theory of this Letter is thus limited to master equations of the form of Eq. (11).

[17] J. Gambetta and H. M. Wiseman, Phys. Rev. A. 68, 062104 (2003). 
[18] H. J. Carmichael, An Open Systems Approach to Quantum Optics (Springer-Verlag, Berlin, 1993).

[19] H. M. Wiseman and L. Diósi, Chem. Phys. 268, 91 (2001); erratum 271, 227 (2001).

[20] C. W. Gardiner, Handbook of Stochastic Methods (Springer, Berlin, 1985).

[21] A. S. Holevo, IEEE Trans. Inf. Theor. IT21 533 (1975); R. Simon, N. Mukunda, and B. Dutta, Phys. Rev. A 49,
1567 (1994).

[22] The quantum version is given in H. M. Wiseman and J. A. Vaccaro, Phys. Rev. Lett. 87, 240402 (2001).

[23] L. Vandenberghe and S. Boyd, Soc. for Indust. and Applied Math. Review 38, 49 (1996).

[24] H. M. Wiseman and G. J. Milburn, Phys. Rev. Lett. 70, 548 (1993); ibid, Phys. Rev. A 49, 1350 (1994). 